\title{
The Treatment of IgA Nephropathy
}

\author{
Kar Neng Lai ${ }^{a, b} \quad$ Joseph C.K. Leung ${ }^{b}$ Sydney C.W. Tang ${ }^{b}$ \\ ${ }^{a}$ Nephrology Center, Hong Kong Sanatorium and Hospital, and bepartment of Medicine, Queen Mary Hospital, \\ University of Hong Kong, Hong Kong, SAR, China
}

\author{
Key Words \\ IgA nephropathy · Treatment · Immunosuppression · \\ Corticosteroid $\cdot$ Renin-angiotensin blockade
}

\begin{abstract}
Background: IgA nephropathy ( $\lg \mathrm{AN}$ ) is a very common glomerulonephritis worldwide. Nevertheless, treatment options for primary IgAN are still largely based on opinion or weak evidence. There is a lack of large randomized controlled trials (RCT) that provide a definitive immunosuppressive protocol for IgAN. The recent KDIGO Clinical Practice Guidelines for Glomerulonephritis have assigned low levels of evidence for almost all recommendations and suggestions related to this nephropathy. Summary: In this article, we review different treatment options and emphasize that the key to therapeutic decision-making is the assessment of an individual's prognosis. The risk of disease progression is closely related to clinical parameters such as proteinuria, hypertension, and impaired glomerular filtration rate. For patients with minor urinary abnormalities, the mainstay of treatment is long-term regular follow-up to detect renal progression and hypertension. Optimized supportive care aiming to maintain proteinuria $<1 \mathrm{~g} /$ day is preferred in the typical patient presenting with microhematuria, significant but nonnephrotic proteinuria, hypertension, and variable degrees of renal failure. The atypical patient with overt nephritic syndrome or rapidly progressive kidney injury that represents a vasculitic form of IgAN should be treated with immunosuppression. Finally, the variant of overlapping syndrome
\end{abstract}

of $\lg \mathrm{AN}$ and lipoid nephrosis that runs a good prognosis should be treated as lipoid nephrosis. Key Message: The treatment of IgAN should be structured according to the clinical scenario.

(c) 2015 S. Karger AG, Basel

\section{Nonimmunosuppressive Treatment}

The mainstay of treatment for IgA nephropathy (IgAN) is the blockade of the renin-angiotensin system. Cheng et al. [1] analyzed 585 patients in 11 randomized controlled trials (RCT), of which 7 trials used placebo/no treatment as controls and 4 trials used other antihypertensive agents as controls. Treatment with angiotensinconverting enzyme inhibitors (ACEI) or angiotensin receptor blockers (ARB) had statistically significant effects on renoprotection and reduction of proteinuria when compared with the control group. A meta-analysis reviewing 6 RCT involving 109 patients showed that the combined treatment with ACEI plus ARB was not more effective than ACR or ARB alone for reducing daily proteinuria [2]. The risk of hyperkalemia was not increased. The long-term effects of combined therapy on renal outcomes remain uncertain. Another meta-analysis of 27 RCT (1,577 participants) using ACEI, ARB or a combination of both versus other antihypertensives, other agents or placebo revealed that renin-angiotensin blockade appears to potentially outweigh the harms in patients with IgAN [3]. The therapeutic effects are predominantly cen- 
tered at a reduction of proteinuria, a surrogate of renal outcome. However, this meta-analysis failed to demonstrate that treatment with any of the antihypertensive agents evaluated affects major renal and/or cardiovascular endpoints or long-term mortality risk beyond the benefit that arises from controlling hypertension. The RCT evidence is insufficiently robust to demonstrate efficacy for any of the other nonimmunosuppressive therapies including fish oil, anticoagulant, and tonsillectomy.

Aliskiren is a relatively new oral direct renin inhibitor (DRI) that has been used for the treatment of hypertension and diabetic nephropathy. Tang et al. [4] recruited 25 consecutive IgAN patients who had persistent proteinuria (protein/creatinine ratio $>113 \mathrm{mg} / \mathrm{mmol}$, which corresponded to proteinuria $1 \mathrm{~g} /$ day) despite receiving the maximum recommended dose of losartan $(100 \mathrm{mg} /$ day $)$ to receive additional DRI treatment. Treatment with aliskiren for 12 months reduced the mean urinary albu$\mathrm{min} /$ creatinine ratio by $26 \%$, with a reduction of $\geq 50 \%$ proteinuria in $24 \%$ of patients. Aliskiren treatment was associated with significant reductions in plasma renin activity, serum interleukin-6, and transforming growth factor- $\beta$. Of note is the detection of transient hyperkalemia in $24 \%$ of patients. Another group of investigators from Hong Kong also studied 22 IgAN patients with persistent proteinuria despite ACEI or ARB treatment [5]. Patients were randomized to either oral aliskiren or placebo for 4 months and then crossed over to the other treatment arm after a washout period. After 4 weeks, there was a significant reduction of proteinuria, which remained low throughout the treatment period. The aliskiren group had a significantly lower proteinuria than the placebo group after 4-16 weeks of treatment. Aliskiren treatment resulted in a modest but significant reduction of the estimated glomerular filtration rate (eGFR) and diastolic blood pressure. Severe hyperkalemia ( $>6$ $\mathrm{mmol} / \mathrm{l}$ ) was not detected in these patients. These two preliminary studies suggest that aliskiren, a DRI, has antiproteinuric effect in IgAN patients who have persistent proteinuria despite ACEI or ARB treatment. Larger RCT are needed to confirm the renoprotective effect of direct renin inhibition.

\section{Immunomodulatory Treatment}

A pathogenetic role for structurally aberrant IgA synthesis is supported by the findings of glomerular deposition of underglycosylated polymeric IgA and the frequently elevated circulating IgA levels in patients with primary IgAN [6]. This, coupled with the recent observation of the role of the ubiquitin-proteasome pathway [7], forms a scientific rationale for applying immunomodulatory agents. Previously, a meta-analysis of 13 RCT involving a total of 623 patients concluded that immunosuppressive agents are a promising strategy and that this approach needed to be investigated further [8]. Research in this area, as in many other renal diseases, is hampered by the slowly progressive nature of the disease, with 10-year renal survival rates exceeding $85 \%$, marked patient heterogeneity, and the lack of a good animal model that closely resembles human IgAN.

\section{Corticosteroids}

A relatively large quantity of data on corticosteroids was contributed by Japanese researchers in early years. Kobayashi et al. [9] reported that steroid treatment in 14 patients with proteinuria 1-2 g/day (versus 29 control subjects) was effective in lowering proteinuria, particularly in patients with better renal function (baseline GFR $>70 \mathrm{ml} / \mathrm{min}$ ). Renal survival after 10 years of follow-up was $80 \%$ in steroid-treated patients versus $34 \%$ in nonsteroid patients [10]. Pozzi et al. [11] reported that steroid treatment in 86 Italian patients for 6 months provided renoprotective effects, and the renal survival was better in the steroid group after 10 years [12]. On the other hand, Lai et al. [13] found no therapeutic value of short-term (6 months) corticosteroid treatment in Chinese IgAN patients with nephrotic range proteinuria. A meta-analysis of 7 RCTs involving 386 subjects suggested that corticosteroids have statistically significant effects on protecting renal function and reducing proteinuria, but gastrointestinal tract reaction is a concern [14]. Another meta-analysis (including 15 controlled, quasi-randomized controlled and noncontrolled trials with 1,542 participants) suggested that corticosteroid therapy is associated with a decrease of proteinuria and with a statistically significant reduction of the risk in end-stage renal failure [15]. Moreover, a subgroup analysis also suggested that long-term steroid therapy had a higher efficiency than standard and short-term therapy. A more recent meta-analysis (including 536 patients who had urinary protein excretion $>1 \mathrm{~g} /$ day and normal renal function from 9 relevant trials) suggested that high-dose and short-term therapy produced significant renal protection, whereas low-dose, long-term steroid use did not [16]. The KDIGO Clinical Practice Guideline for Glomerulonephritis suggested that there is low-quality evidence for corticosteroids to provide additional benefits on top of optimized supportive care [17]. The KDIGO group recommended that patients with per- 
sistent proteinuria $>1 \mathrm{~g} /$ day despite adequate ACEI or ARB, blood pressure control, and GFR $>50 \mathrm{ml} / \mathrm{min} / 1.73$ $\mathrm{m}^{2}$ may receive a 6-month course of steroid therapy. A multicenter clinical trial aimed at defining the efficacy and safety of steroid treatment in IgAN is currently underway.

Recently, a European consortium (VALIGA study) has examined 1,147 patients encompassing the whole spectrum of IgAN from 13 countries [18]. Over a median follow-up of 4.7 years, $86 \%$ received renin-angiotensin system blockade and $42 \%$ received corticosteroid or immunosuppressive drugs. The renal pathology was classified according to the Oxford classification of IgAN [19], and the independent predictive value of the pathology MEST score was reduced by corticosteroid or immunosuppressive therapy. A retrospective analysis of a subgroup of patients receiving corticosteroid plus renin-angiotensin system blockade comparing match patients receiving ACEI/ARB alone demonstrated a reduction of proteinuria and the rate of renal function decline [20]. These benefits extended to patients with an eGFR $\leq 50$ $\mathrm{ml} / \mathrm{min} / 1.73 \mathrm{~m}^{2}$, and the effect increased proportionally with the severity of proteinuria.

\section{Cyclophosphamide in Combination with \\ Corticosteroids}

Evidence that pulse corticosteroid plus intravenous or oral cyclophosphamide slow the progression of advanced IgAN was provided by several groups worldwide.

In 38 patients with progressive renal deterioration, Ballardie and Roberts [21] showed that 5-year renal survival was considerably better in cyclophosphamide-treated patients than in controls at five years (72 vs. 6\%). Proteinuria and erythrocyturia were reduced after 12 and 6 months of treatment, respectively. This study may be faulted, however, for suboptimal blood pressure control and insufficient use of medications that block the reninangiotensin system, the unusually poor survival rate of the placebo group, and the small number of patients. In another prospective, uncontrolled, open-label trial, 12 patients with crescentic IgAN received three doses of methylprednisolone at $15 \mathrm{mg} / \mathrm{kg} /$ day, followed by intravenous cyclophosphamide at $0.5 \mathrm{~g} / \mathrm{m}^{2} /$ month for 6 months [22]. Serum creatinine improved with reduction of proteinuria, suggesting a beneficial role of cyclophosphamide in crescentic glomerulonephritis. In another observational, uncontrolled study, similar results were reported in 21 patients with advanced IgAN treated with intravenous pulse cyclophosphamide $\left(0.75 \mathrm{~g} / \mathrm{m}^{2} /\right.$ month $)$ for 6 months together with low-dose oral prednisolone
[23]. Mitsuiki et al. [24] retrospectively examined the outcome of 35 patients with histologically advanced IgAN, of whom 27 received prednisolone for 2 years and oral cyclophosphamide (50 mg/day) for 6 months, and the remaining 8 received supportive treatment. Renal prognosis was significantly better in the treatment group.

Overall, these studies suggest that combined cyclophosphamide/steroid therapy may benefit patients at a very high risk of renal failure, namely those with a progressive decline in GFR and/or crescentic lesions. Due to the side effects, it is reasonable to use short-term cyclophosphamide with corticosteroids for IgAN patients with true crescentic or rapidly progressive glomerulonephritis. The KDIGO Clinical Practice Guideline for Glomerulonephritis suggested a similar approach (low-quality evidence) [19].

\section{Tonsillectomy in Combination with Corticosteroids}

For a long time, tonsillectomy was considered a treatment option for IgAN, aimed at removing a relevant source of pathogens, which can multiply in tonsil crypts as well as in macrophages and B-cells in lymphoid tonsil follicles. This specific antigen challenge was thought to induce a supernormal IgA synthesis, as tonsil lymphocytes from IgAN patients showed a higher production of dimeric and undergalactosylated IgA1 than control subjects. In Japan, tonsillectomy/pulse steroid therapy has frequently been used for treatment of early IgAN with favorable outcomes [25]. A recent meta-analysis of 7 nonrandomized studies ( 6 from Japan and 1 from China) comprising 858 patients (534 underwent tonsillectomy and 324 did not) has shown that tonsillectomy combined with either normal steroid or pulse steroid treatment, but not tonsillectomy or steroid treatment alone, resulted in higher remission rates with favorable long-term efficacy at both 5- and 10-year follow-up [26].

Outside of Japan, the benefits of tonsillectomy have been largely less impressive than expected. A retrospective review of 61 Caucasian patients showed that tonsillectomy did not alter the rate of disease progression after 20 years of follow-up [27]. Recently, a Hungarian study comparing 166 IgAN patients who did not undergo tonsillectomy with 98 patients who did undergo tonsillectomy has suggested that tonsillectomy may delay the renal progression especially in patients with macrohematuria [28]. An intriguing finding is that decreased serum galactose-deficient IgA1 after tonsillectomy indicates that the palatine tonsils are probably a major site of galactose-deficient IgA1-producing cells [29]. Yet, the clinical efficacy of tonsillectomy in IgAN is completely demolished by 
two latest studies. Sato et al. [30] found that a preoperative tonsillectomy did not affect the recurrence of IgAN in renal allograft. More importantly, the first national multicenter RCT from Japan failed to demonstrate any beneficial effect of tonsillectomy combined with pulse steroid therapy over pulse steroid therapy alone [31]. In Chinese patients, tonsillectomy did not independently correlate with clinical remission nor did it improve renal survival among a case cohort of 112 patients [32]. The 2010 KDIGO Clinical Practice Guideline for Glomerulonephritis suggested that tonsillectomy should not be performed for IgAN (low-quality evidence) [19].

\section{Calcineurin Inhibitors}

Earlier experience of cyclosporine use in IgAN was less favorable. Lai et al. [33] conducted a randomized prospective single-blind study of 19 patients with proteinuria $>1.5 \mathrm{~g} /$ day. Patients who received cyclosporine had a significant reduction of proteinuria, serum IgA, and an increase of plasma albumin concentration compared with the placebo patients. However, there was a transient deterioration of renal function during treatment despite within-range trough drug levels. Two recent short-term studies from China provided additional information on cyclosporine A therapy. In the first study, patients were treated with prednisone $60 \mathrm{mg} /$ day or cyclosporine A $3 \mathrm{mg} / \mathrm{kg} /$ day plus prednisone $40 \mathrm{mg} /$ day [34]. Patients receiving combined therapy had a greater reduction of proteinuria than the steroid group. Combined therapy led to a higher remission rate in patients with pathology of lesser severity. The second study also compared the clinical efficacy between steroid alone and medium-dose cyclosporine plus steroid therapy [35]. After 12 months of treatment, all patients in the combined group and $87.5 \%$ of patients in the steroid-alone group had a $\geq 50 \%$ reduction of proteinuria. However, $8.7 \%$ of patients in the combined group had a $50 \%$ increase in the baseline serum creatinine level, while none did in the steroid alone group. Moreover, $8.33 \%$ of patients in the combined group developed severe infection during treatment. These data discourage the indiscriminate use of cyclosporine in IgAN due to potential nephrotoxicity.

Data on the other calcineurin inhibitors, such as tacrolimus, are sparse. Zhang et al. [36] reported inducing a remission of proteinuria in 14 patients with refractory IgAN by tacrolimus. These investigators suggested the therapeutic mechanism possibly mediated via stabilizing podocytic cytoskeleton. Kim et al. [37] demonstrated that tacrolimus effectively reduced proteinuria in IgAN patients with normal blood pressure and suggested tacroli- mus as an alternative to corticosteroid and ACEI/ARB for patients unable to endure anti-hypertensive medication.

\section{Azathioprine}

A retrospective analysis of 74 IgAN patients followed up for 10 years showed that long-term azathioprine combined with low-dose prednisone did not alter the clinical course compared to that of untreated controls [38]. However, in a subgroup of patients with heavy proteinuria $>3$ $\mathrm{g} /$ day and baseline serum creatinine $1.4-2.5 \mathrm{mg} / \mathrm{dl}$, this immunosuppressive regimen reduced the risk of doubling serum creatinine compared to controls ( 27 vs. $78 \%$ ) and delayed the progression to end-stage renal failure (17 vs. 55\%). The Japanese Paediatric IgA Nephropathy Treatment Study Group randomized 78 children with newly diagnosed early IgAN to either receive prednisolone, azathioprine, heparin-warfarin, and dipyridamole or the combination of heparin-warfarin and dipyridamole only [39]. The inclusion of azathioprine reduced the urinary protein and serum IgA. The study was flawed by a lack of data on baseline proteinuria and creatinine clearance as well as blood pressure control in both groups. An Italian prospective randomized study of 207 subjects showed that the addition of azathioprine to corticosteroids did not provide additional benefits in terms of renal survival compared to corticosteroids alone in patients with proteinuria $\geq 1 \mathrm{~g} /$ day and plasma creatinine $\leq 2.0 \mathrm{mg} / \mathrm{dl}$ [40]. Intriguingly, in the same cohort of patients, these investigators studied patients with plasma creatinine $\geq 2.0 \mathrm{mg} / \mathrm{dl}$ [41]. Six-year renal survival was similar in the two groups. In Cox analysis, the addition of azathioprine may be slightly more effective than corticosteroids alone in patients with chronic renal insufficiency, although with an increase of side effects. Current data therefore suggest that the addition of azathioprine was of limited therapeutic advantage and may even be potentially toxic.

\section{Mycophenolate Mofetil}

To date, 6 randomized clinical trials have been published on the use of MMF in IgAN, which add more controversy than consensus. Although these trials have produced conflicting results, they differ significantly in patient selection and treatment duration and deserve attention.

The first randomized study was conducted in $62 \mathrm{Chi}-$ nese patients with severe IgAN and urinary protein $>2.0$ $\mathrm{g} /$ day who received MMF or oral prednisone for at least 12 months [42]. After 18 months of follow-up, the MMF group showed a significant improvement in proteinuria and serum lipids compared to the prednisone group. In 
another study of 34 Belgian patients with impaired renal function, histologically unfavorable criteria and arterial hypertension, after instituting salt restriction and ACEI therapy in all, MMF failed to demonstrate a better beneficial effect after 3 years of evaluation [43]. In a similar study that recruited patients with even more advanced renal insufficiency using MMF as a 'salvage' therapy, a worse outcome occurred in the MMF group [44].

Tang et al. [45] treated 40 Chinese patients with mild tubulointerstitial lesions with persistent proteinuria $>1$ $\mathrm{g} /$ day despite full angiotensin blockade for 6 months. Twelve months after stopping MMF, the overall remission rate was significantly higher in MMF-treated patients whose proteinuria dropped to $62 \%$ of baseline, whereas urinary protein in control patients increased to $120 \%$ of baseline. Serum interleukin- 6 levels and, more intriguingly, in vitro binding of IgA to mesangial cells were elevated at baseline in both groups compared with normal healthy subjects; however, after MMF treatment, these parameters were comparable to those of the healthy subjects. Serum interleukin-6 concentration and mesangial binding of IgA in patients who did not receive MMF showed no change. After 6 years of follow-up, the difference in proteinuria between the two groups was lost, but renal survival was significantly better in the MMF group [46]. In another Italian study, a subset of IgAN patients with florid glomerular changes treated with MMF and steroid showed remission of proteinuria and reversal of progressive renal failure [47]. A recent study from China compared between combined therapy with MMF and prednisone and combined therapy of cyclophosphamide and prednisone for severe IgAN [48]. The former achieved a higher remission rate with better reduction of proteinuria and improvement of renal function. Importantly, the incidence of adverse effects was significantly lower in the MMF group compared to the cyclophosphamide group.

Overall, MMF appears to be effective in reducing proteinuria in Chinese but not necessarily in Caucasian subjects. Therefore, ethnic differences may be one possible reason to account for the differences observed in these studies. Another possibility is the mild histologic grade in Tang's study [45] compared to the moderate-to-severe grades in the studies by Maes et al. [43] and Frisch et al. [44], despite more favorable results in patients with severe IgAN from Italy and China. It is interesting to point out that MMF may ameliorate IgAN through other mechanisms than by reducing interleukin- 6 production and mesangial binding of IgA [45]. A recent study showed that mycophenolic acid (derived from MMF) can up-reg-

Treatment of IgA Nephropathy ulate the core $1 \beta 3$-Gal-T-specific molecular chaperone (Cosmc) expression and hence reverse the IgA1 aberrant $\mathrm{O}$-glycosylation level in peripheral lymphocytes of patients with IgAN [49]. Both impaired Cosmc expression and defective IgA1 aberrant O-glycosylation play a pivotal role in the pathogenesis of IgAN [50].

Further observation and studies are needed to provide more definitive answers on the efficacy of MMF in IgAN. The KDIGO Clinical Practice Guideline for Glomerulonephritis (2012) suggested not using MMF in IgAN (lowquality evidence) [19].

\section{Other Therapies}

In vitro study showed that peroxisome proliferatoractivated receptor- $\gamma$ (PPAR- $\gamma$ ) agonist attenuates inflammatory response in activated tubular epithelial cells in IgAN by down-regulating the expression of angiotensin receptor subtype-1 [51]. Dual treatment of PPAR- $\gamma$ and ARB provide a synergistic effect in reducing inflammation and angiotensin II signaling in renal tubular epithelial cells, and the therapeutic benefit was confirmed in an animal model of IgAN [52].

Enteric budesonide targeted to Peyer's patches in the ileocecal region has been shown to reduce proteinuria by $23 \%$ and modestly augment eGFR by $8 \%$ in 16 patients who received this new formulation for 6 months followed by 3 months of further observation [53]. Based on these encouraging results, a multicenter phase IIb trial is currently being planned in Europe.

The renoprotective pleiotropic effect of 3-hydroxy3-methylglutaryl coenzyme reductase inhibitors (statins) was studied in 24 IgAN patients who did not receive corticosteroids or immunosuppressive agents [54]. After 1 year of statin treatment, there was an $8 \%$ increase in eGFR despite no significant reduction of proteinuria.

Complement activation plays a role in the pathogenesis of IgAN involving the alternative and lectin pathways [55]. Rosenblad et al. [56] administered eculizumab (antiC5) to an IgAN patient with rapidly progressive disease leading to renal failure refractory to immunosuppressive therapy. Treatment led to a stabilization of renal function and reduced proteinuria. Renal function deteriorated rapidly following the discontinuation of therapy and was temporarily restored after a single dose of eculizumab.

The potential role of the spleen tyrosine kinase (SYK), an intracellular protein tyrosine kinase involved in cell signaling downstream of immunoreceptors, in IgAN is under investigation. Increased expression of total and 


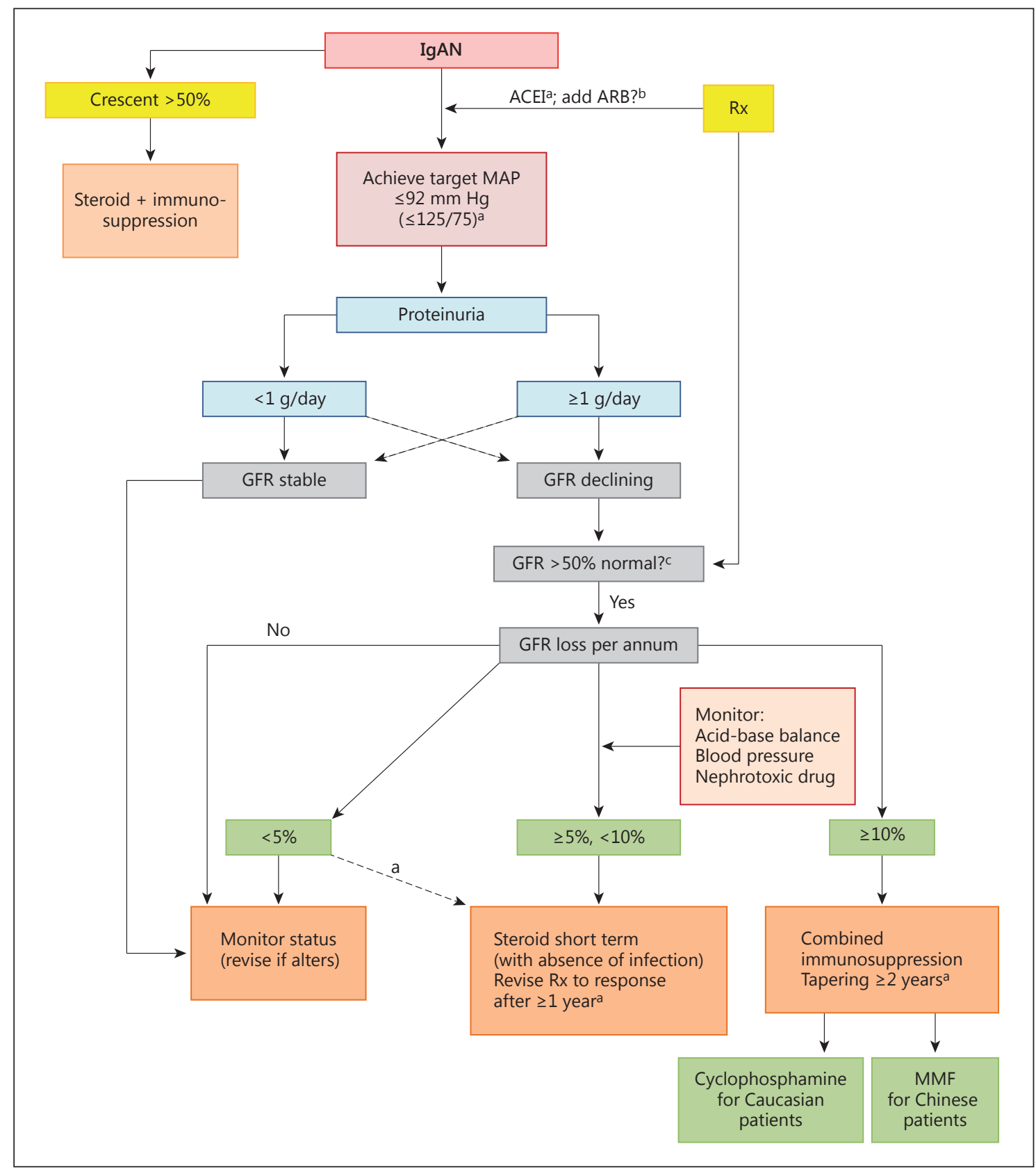

Fig. 1. An algorithm of recommended treatment options for IgAN. Modified from Lai [60] and used with permission. Broken lines denote less frequently encountered scenarios. $\mathrm{Rx}=$ Treatment. ${ }^{\text {a }}$ Therapy with efficacy-ofevidence base grade 1 data. ${ }^{\mathrm{b}}$ Therapy with high a priori evidence to use but not tested independently in RCT. ${ }^{\mathrm{c}}$ There is no evidence that immunosuppressive drugs can benefit declining function in patients starting therapy with $>50 \%$ loss in GFR.

phosphorylated SYK has been observed in renal biopsies of patients with IgAN, and pharmacological inhibition of SYK or its knockdown by siRNA significantly reduced proliferation and the synthesis of proinflammatory mediators in human mesangial cells exposed to IgA1 isolated from patients with IgAN and inhibited mesangial cell proliferation [57]. An RCT examining the clinical utility of fostamatinib (a selective oral SYK inhibitor) in patients with IgAN is currently ongoing. 


\section{Recommendations for Different Clinical Scenarios}

\section{Rapidly Progressive/Vasculitic Form}

This is characterized by a short history and rapidly deteriorating renal function. Renal biopsy shows crescent formation in $>50 \%$ of glomeruli. Chronic tubulointerstitial atrophy and fibrosis are not prominent despite mononuclear cell infiltration. It is reasonable to use pulse steroid followed by oral steroid plus short-term intravenous/oral cyclophosphamide.

\section{Overlapping Syndrome of IgAN and Lipoid \\ Nephrosis}

This is characterized by a short history of nephrotic syndrome and normally deteriorating renal function. Renal biopsy shows minimal glomerular and tubular pathology except mesangial deposits of IgA. These patients should be treated as lipoid nephrosis patients, with corticosteroid and gentle dose reduction [58]. The efficacy and safety of such an approach was confirmed in a recent cohort of Chinese subjects [59]. The clinical picture behaves like lipoid nephrosis with relapses but chronic renal impairment is uncommon.

\section{Typical Patient Presenting with Microhematuria,} Significant but Nonnephrotic Proteinuria, Hypertension, and Variable Degrees of Renal Failure

For those patients with proteinuria $<1 \mathrm{~g} /$ day, stable renal function (eGFR $>60 \mathrm{ml} / \mathrm{min} / 1.73 \mathrm{~m}^{2}$ ), and normotensive (blood pressure $<125 / 75 \mathrm{~mm} \mathrm{Hg}$ ), the mainstay of treatment is long-term regular follow-up to detect renal progression and hypertension. Optimized supportive care aiming at maintaining proteinuria $<1 \mathrm{~g} /$ day is preferred

A treatment algorithm according to the clinical status is outlined in figure 1 .

\section{Acknowledgement}

Part of the works cited in this review was supported by the L\&T Charitable Foundation and the House of INDOCAFE.

\section{Disclosure Statement}

The authors have no conflicts of interest to declare.

\section{References}

1 Cheng J, Zhang W, Zhang XH, He Q, Tao XJ, Chen JH: ACEI/ARB therapy for IgA nephropathy: a meta analysis of randomised controlled trials. Int J Clin Pract 2009;63:880888.

2 Cheng J, Zhang X, Tian J, Li Q, Chen J: Combination therapy an ACE inhibitor and an angiotensin receptor blocker for IgA nephropathy: a meta-analysis. Int J Clin Pract 2012;66: 917-923.

3 Reid S, Cawthon PM, Craig JC, Samuels JA, Molony DA, Strippoli GF: Non-immunosuppressive treatment for IgA nephropathy. Cochrane Database Syst Rev 2011;CD003962.

-4 Tang SC, Lin M, Tam S, Au WS, Ma MK Yap DY, Ho YW, Lai KN: Aliskiren combined with losartan in immunoglobulin A nephropathy: an open-labeled pilot study. Nephrol Dial Transplant 2012;27; 613-618.

-5 Szeto CC, Kwan BC, Chow KM, Leung CB, Li PK: The safety and short-term efficacy of aliskiren in the treatment of immunoglobulin A nephropathy - a randomized cross-over study. PLoS One 2013;8:e62736.

6 Lai KN, To WY, Li PK, Leung JC: Increased binding of polymeric lambda-IgA to cultured human mesangial cells in IgA nephropathy. Kidney Int 1996;49:839-845.

7 Tang SC, Lai KN: The ubiquitin-proteasome pathway and IgA nephropathy: a novel link? Kidney Int 2009;75:457-459.
8 Samuels JA, Strippoli GF, Craig JC, Schena FP, Molony DA: Immunosuppressive treatments for immunoglobulin A nephropathy: a meta-analysis of randomized controlled trials. Nephrology (Carlton) 2004;9:177-185.

9 Kobayashi Y, Fujii K, Hiki Y, Tateno S: Steroid therapy in IgA nephropathy: a prospective pilot study in moderate proteinuric cases. Q J Med 1986;61:935-943.

10 Kobayashi Y, Hiki Y, Kokubo T, Horii A, Tateno S: Steroid therapy during the early stage of progressive IgA nephropathy. A 10-year follow-up study. Nephron 1996;72:237-242.

-11 Pozzi C, Bolasco PG, Fogazzi GB, Andrulli S, Altieri P, Ponticelli C, Locatelli F: Corticosteroids in IgA nephropathy: a randomised controlled trial. Lancet 1999;353:883-887.

- 12 Pozzi C, Andrulli S, Del Vecchio L, Melis P, Fogazzi GB, Altieri P, Ponticelli C, Locatelli F: Corticosteroid effectiveness in IgA nephropathy: long-term results of a randomized, controlled trial. J Am Soc Nephrol 2004;15:157-163.

-13 Lai KN, Lai FM, Ho CP, Chan KW: Corticosteroid therapy in IgA nephropathy with nephrotic syndrome: a long-term controlled trial. Clin Nephrol 1986;26:174-180.

14 Cheng J, Zhang X, Zhang W, He Q, Tao X, Chen J: Efficacy and safety of glucocorticoids therapy for IgA nephropathy: a meta-analysis of randomized controlled trials. Am J Nephrol 2009;30:315-322.
15 Zhou YH, Tang LG, Guo SL, Jin ZC, Wu MJ, Zang JJ, Xu JF, Wu CF, Qin YY, Cai Q, Gao $\mathrm{QB}$, Zhang SS, Yu DH, He J: Steroids in the treatment of IgA nephropathy to the improvement of renal survival: a systematic review and meta-analysis. PLoS One 2011;6:e18788.

16 Lv J, Xu D, Perkovic V, Ma X, Johnson DW, Woodward M, Levin A, Zhang H, Wang H; TESTING Study Group: Corticosteroid therapy in IgA nephropathy. J Am Soc Nephrol 2012;23:1108-1116.

17 Radhakrishnan J, Cattran DC: The KDIGO practice guideline on glomerulonephritis: reading between the (guide)lines - application to the individual patient. Kidney Int 2012;82:840-856.

18 Coppo R, Troyanov S, Bellur S, et al: Validation of the Oxford classification of IgA nephropathy in cohorts with different presentations and treatments. Kidney Int 2014;86: 826-836.

19 Working Group of the International IgA Nephropathy Network and the Renal Pathology Society: The Oxford classification of IgA nephropathy: pathology definitions, correlations, and reproducibility. Kidney Int 2009; 76:546-556.

20 Tesar V, Troyanov S, Bellur S, et al: Corticosteroid in IgA nephropathy: a retrospective analysis from the VALIGA study. J Am Soc Nephrol 2015, Epub ahead of print. 
21 Ballardie FW, Roberts IS: Controlled prospective trial of prednisolone and cytotoxics in progressive IgA nephropathy. J Am Soc Nephrol 2002;13:142-148.

22 Tumlin JA, Lohavichan V, Hennigar R: Crescentic, proliferative IgA nephropathy: clinical and histological response to methylprednisolone and intravenous cyclophosphamide. Nephrol Dial Transplant 2003;18:1321-1329.

23 Rasche FM, Klotz CH, Czock D, Karges W, Muche R, Jehle PM, Mertz A, Keller F: Cyclophosphamide pulse therapy in advanced progressive IgA nephropathy. Nephron Clin Pract 2003;93:c131-c136.

24 Mitsuiki K, Harada A, Okura T, Higaki J: Histologically advanced IgA nephropathy treated successfully with prednisolone and cyclophosphamide. Clin Exp Nephrol 2007;11: 297-303.

25 Moriyama T, Nitta K: Tonsillectomy and steroid pulse therapy for IgA nephropathy. Tohoku J Exp Med 2011;224:243-250.

-26 Wang Y, Chen J, Wang Y, Chen Y, Wang L, Lv Y: A meta-analysis of the clinical remission rate and long-term efficacy of tonsillectomy in patients with IgA nephropathy. Nephrol Dial Transplant 2011;26:1923-1931.

27 Piccoli A, Codognotto M, Tabbi MG, Favaro E, Rossi B: Influence of tonsillectomy on the progression of mesangioproliferative glomerulonephritis. Nephrol Dial Transplant 2010 25:2583-2589.

28 Kovacs T, Vas T, Kovesdy CP, Degrell P, Nagy G, Rekasi Z, Wittmann I, Nagy J: Effect of tonsillectomy and its timing on renal outcomes in Caucasian IgA nephropathy patients. Int Urol Nephrol 2014;46:2175-2182.

29 Nakata J, Suzuki Y, Suzuki H, Sato D, Kano T, Yanagawa H, Matsuzaki K, Horikoshi S, Novak J, Tomino Y: Changes in nephritogenic serum galactose-deficient IgAl in IgA nephropathy following tonsillectomy and steroid therapy. PLoS One 2014;9:e89707.

-30 Sato Y, Ishida H, Shimizu T, Tanabe K: Evaluation of tonsillectomy before kidney transplantation in patients with IgA nephropathy. Transpl Immunol 2014;30:12-17.

-31 Kawamura T, Yoshimura M, Miyazaki Y, et al: A multicenter randomized controlled trial of tonsillectomy combined with steroid pulse therapy in patients with immunoglobulin A nephropathy. Nephrol Dial Transpl 2014;29: 1546-1553.

32 Chen Y, Tang Z, Wang Q, Yu Y, Zeng C, Chen H, Liu ZH, Li LS: Long-term efficacy of tonsillectomy in Chinese patients with IgA nephropathy. Am J Nephrol 2007;27:170-175.

33 Lai KN, Lai FM, Li PK, Vallance-Owen J: Cyclosporin treatment of $\operatorname{IgA}$ nephropathy: a short term controlled trial. Br Med J 1987;295: $1165-1168$.

34 Liu H, Xu X, Fang Y, Ji J, Zhang X, Yuan M, Liu C, Ding X: Comparison of glucocorticoids alone and combined with cycloporine $\mathrm{A}$ in patients with IgA nephropathy: a prospective randomized controlled trial. Intern Med 2014;53:675-681.
35 Xu L, Liu ZC, Guan GJ, Lv XA, Luo Q: Cyclosporine A combined with medium/low dose prednisone in progressive IgA nephropathy. Kaohsiung J Med Sci 2014;30:390-395.

36 Zhang Q, Shi SF, Zhu L, Lv JC, Liu L, Chen Y, Zhang $\mathrm{H}$, Wang $\mathrm{H}$ : Tacrolimus improves the proteinuria remission in patients with refractory IgA nephropathy. Am J Nephrol 2012;35: 312-320.

37 Kim YC, Chin HJ, Koo HS, Kim S: Tacrolimus decreases albuminuria in patients with IgA nephropathy and normal blood pressure: a double-blind randomized controlled trial of efficacy of tacrolimus on IgA nephropathy. PLoS One 2013;8:e71545.

38 Goumenos DS, Davlouros P, El Nahas AM, Ahuja M, Shortland JR, Vlachojannis JG, Brown CB: Prednisolone and azathioprine in IgA nephropathy - a ten-year follow-up study. Nephron Clin Pract 2003;93:C58-C68.

39 Yoshikawa N, Ito H: Combined therapy with prednisolone, azathioprine, heparin-warfarin, and dipyridamole for paediatric patients with severe IgA nephropathy - is it relevant for adult patients? Nephrol Dial Transplant 1999;14:1097-1099.

40 Pozzi C, Andrulli S, Pani A, Del Vecchio L, Fogazzi G, Vogt B, De Cristofaro V, Allegri L, Cirami L, Procaccini AD, Locatelli F: Addition of azathioprine to corticosteroids does not benefit patients with IgA nephropathy. J Am Soc Nephrol 2010;21:1783-1790.

41 Pozzi C, Andrulli S, Pani A, Scaini P, Roccatello D, Fogazzi G, Pecchini P, Rustichelli R, Finocchiaro P, Del Vecchio L, Locatelli F: IgA nephropathy with severe chronic renal failure: a randomized controlled trial of corticosteroid and azathioprine. J Nephrol 2013;26: 86-93.

42 Chen X, Chen P, Cai G, Wu J, Cui Y, Zhang Y, Liu S, Tang L: A randomized control trial of mycophenolate mofeil treatment in severe IgA nephropathy (in Chinese). Zhonghua Yi Xue Za Zhi 2002;82:796-801.

43 Maes BD, Oyen R, Claes K, Evenepoel P, Kuypers D, Vanwalleghem J, Van Damme B, Vanrenterghem YF: Mycophenolate mofetil in IgA nephropathy: results of a 3-year prospective placebo-controlled randomized study. Kidney Int 2004;65:1842-1849.

44 Frisch G, Lin J, Rosenstock J, Markowitz G, D’Agati V, Radhakrishnan J, Preddie D, Crew J, Valeri A, Appel G: Mycophenolate mofetil (MMF) vs placebo in patients with moderately advanced IgA nephropathy: a double-blind randomized controlled trial. Nephrol Dial Transplant 2005;20:2139-2145.

45 Tang S, Leung JC, Chan LY, Lui YH, Tang CS, Kan CH, Ho YW, Lai KN: Mycophenolate mofetil alleviates persistent proteinuria in IgA nephropathy. Kidney Int 2005;68:802-812.

46 Tang SC, Tang AW, Wong SS, Leung JC, Ho YW, Lai KN: Long-term study of mycophenolate mofetil treatment in IgA nephropathy. Kidney Int 2010;77:543-549.
47 Roccatello D, Rossi D, Marletto F, Naretto C, Sciascia S, Baldovino S, Piras D, Giachino O: Long-term effects of methylprednisolone pulses and mycophenolate mofetil in IgA nephropathy patients at risk of progression. J Nephrol 2012;25:198-203.

- 48 Liu X, Dewei D, Sun S, Xu G, Liu H, He L, Zhang P: Treatment of severe IgA nephropathy: mycophenolate mofetil/prednisone compared to cyclophosphamide/ prednisone. Int J Clin Pharmacol Ther 2014;52:95-102.

-49 Xie L, Tan C, Fan J, Fu P, Tang Y, Tao Y, Qin W: Mycophenolic acid reverses IgA1 aberrant glycosylation through up-regulating Cosmc expression in IgA nephropathy. Int Urol Nephrol 2013;45:571-579.

50 Lai KN: Pathogenesis of IgA nephropathy. Nat Rev Nephrol 2012;8:275-283.

51 Xiao J, Leung JC, Chan LY, Tang SC, Lai KN: Crosstalk between peroxisome proliferatoractivated receptor-gamma and angiotensin II in renal tubular epithelial cells in IgA nephropathy. Clin Immunol 2009;132:266-276.

52 Lai KN, Chan LY, Guo H, Tang SC, Leung JC: Additive effect of PPAR-gamma agonist and ARB in treatment of experimental IgA nephropathy. Pediatr Nephrol 2011;26:257266.

53 Smerud HK, Barany P, Lindstrom K, Fernström A, Sandell A, Påhlsson P, Fellström B: New treatment of IgA nephropathy: enteric budesonide targeted to the ileocecal region ameliorates proteinuria. Nephrol Dial Transplant 2011;26:3237-3242.

54 Moriyama T, Oshima Y, Tanaka K, Iwasaki C, Ochi A, Itabashi M, Takei T, Uchida K, Nitta $\mathrm{K}$ : Statins stablize the renal function of IgA nephropathy. Ren Fail 2014;36:356-360.

55 Maillard N, Wyatt RJ, Julian BA, Kiryluk K, Gharavi A, Fremeaux-Bacchi V, Novak J: Current understanding of the role of complement in IgA nephropathy. J Am Soc Nephrol 2015, Epub ahead of print.

-56 Rosenblad T Rebetz J, Johansson M, Bekassy Z, Sartz L, Karpman D: Eculizumab treatment for rescue of renal function in IgA nephropathy. Pediatr Nephrol 2014;29:2225-2228.

57 Kim MJ, McDaid JP, McAdoo SP, Barratt J, Molyneux K, Masuda ES, Pusey CD, Tam FW: Spleen tyrosine kinase is important in the production of proinflammatory cytokines and cell proliferation in human mesangial cells following stimulation with IgA1 isolated from IgA nephropathy patients. J Immunol 2012;189:3751-3758.

58 Lai KN, Lai FM, Chan KW, Ho CP, Leung AC, Vallance-Owen J: An overlapping syndrome of IgA nephropathy and lipoid nephrosis. Am J Clin Pathol 1986;86:716-723.

59 Wang J, Juan C, Huang Q, Zeng C, Liu Z: Corticosteroid therapy in IgA nephropathy with minimal change-like lesions: a single-centre cohort study. Nephrol Dial Transplant 2013; 28:2339-2345.

60 Lai KN (ed): A Practical Manual of Renal Medicine. Hong Kong, World Scientific, 2009, pp 115-119. 